\title{
Auditory Brainstem Response Measurements in Newborns: Which Electrode Placement Is Better?
}

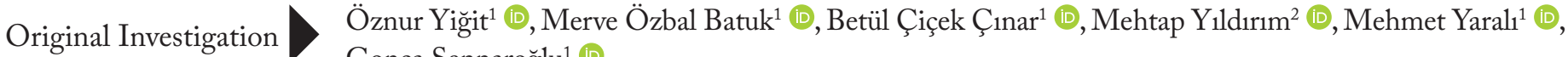 \\ Gonca Sennaroğlu' ${ }^{1}$ \\ ${ }^{1}$ Department of Audiology, Hacettepe University, School of Health Sciences, Ankara, Turkey \\ ${ }^{2}$ Unit of Audiology and Speech Pathology, Hacettepe University Hospital, Ankara, Turkey
}

Abstract

ORCID iDs of the authors: Ö.Y. 0000-0003-4577-1055; M.0̈.B. 0000-0003-4771-8127 B.C.C. .0000-0001-5496-3708; M.Y. 0000-0001-6756-9662; M. Yaralı 0000-0003-1870-0702 G.S. $0000-0002-3337-2391$.

Cite this article as: Yiğit Ö, Özbal Batuk $M$, Çiçek Çınar B, Yıldırım M, Yaralı M, Sennaroğlu G. Auditory Brainstem Response Measurements in Newborns: Which Electrode Placement Is Better? Turk Arch Otorhinolaryngol 2020; 58(2): 112-7.

Corresponding Author: Öznur Yiğit, ozyidu@gmail.com Received Date: 03.01 .2020 Accepted Date: 28.04.2020

Content of this journal is licensed under a Creative Commons Attribution 4.0 International License. Available online at www.turkarchotolaryngol.net
Objective: The objective of this study was to determine the fastest and the most effective auditory brainstem response (ABR) measurement protocol for audiological diagnosis in babies up to three months of age.

Methods: Twenty-two newborns (aged 0 to 63 days) who passed the newborn screening test in at least one ear were evaluated in the study. The ABR were recorded with click stimulus using two different electrode montages ( $1^{\text {st }}$ montage: ipsilateral mastoid, contralateral mastoid, vertex. $2^{\text {nd }}$ montage: nape of the neck, vertex, cheek). Latencies of waves I, III, V and duration of the test were recorded and analyzed.

Results: Wave V latencies from both electrode montages were statistically shortest at the level of $70 \mathrm{~dB}$ $\mathrm{nHL}$ and longest at the level of $20 \mathrm{dBnHL}(\mathrm{p}=0.00)$. When the duration of the test at three different inten- sity levels were compared between the two electrode montages, only the test durations at $50 \mathrm{dBnHL}$ were significantly different $(p=0.017)$. The test times at 70 $\mathrm{dBnHL}$ in the first montage were observed to be significantly different in babies aged 1 to 30 days and aged 31 to 63 days ( $p=0.005)$.

Conclusion: In protocols to evaluate the hearing of pediatric groups, it is very important to complete the $\mathrm{ABR}$, which has significant value in early diagnosis, in a short time and reliably. It is concluded that in terms of practicality, the second montage is more advantageous and comfortable for both audiologists and newborns in single channel ABR systems.

Keywords: Hearing loss, newborn hearing screening, auditory brainstem response, electrode montage, click stimulus

\section{Introduction}

Screening procedures are performed to detect a certain disorder in a target population before symptoms become evident $(1,2)$. In that regard, the aim of hearing screenings is to identify the children suspected of hearing loss and refer them to advanced differential diagnosis tests as necessary (1-3). It is imperative that all babies undergo hearing screening in their first month of life; and diagnostic evaluation should be performed in the first three months in all babies that fail the initial screening regardless of whether or not they have any risk. Further, the babies diagnosed with a hearing problem must be provided with the necessary amplification and educational support in their first three to six months of life. The success of screening depends on the early diagnosis of the hearing loss and on performing the tests in a swift manner (4).
To diagnose hearing loss in babies under the age of one year, otoacoustic emission (OAE) and auditory brainstem response (ABR) tests, which are the objective tests based on physiological response, must be included in the test battery $(5,6)$. Since the devices used in ABR tests for scanning purposes can test only at $35 \mathrm{dBnHL}$, diagnostic devices should be able to perform tests at higher dBnHL levels (3).

Audio-stimulated potentials (ASP) are the electrophysiological responses stemming from audio or electrical stimuli and auditory nerves, brainstem, or neural activities at the cortical level. Electrophysiological responses are recorded with the electrodes placed on the skull (7). It is reported in the literature that the highest amplitude waves in the ABR test are obtained by placing 
the electrodes on the vertex, the forehead and the nape (8). To minimize patient-related artefacts that might occur during the ABR test, the babies need to be in their natural sleep for at least 45 minutes. Therefore, it is crucial to perform the test when babies are in their natural sleep and in a single session to save time (8). Considering all these factors, it can be said that in order to ensure early diagnosis, the devices to be used must be compatible and equipped with different test modules (e.g., $\mathrm{OAE}$ and $\mathrm{ABR}$ ), so that the tests can be performed swiftly and the diagnosis of a potential hearing loss can be made in due time $(9,10)$.

The objective of this study was to compare ABR waves in two different electrode montages by using a click stimulus, to determine if a more reliable ABR recording at a stimulus intensity close to the newborns' hearing threshold is possible, and to investigate which electrode array must be used for a more reliable and faster newborn hearing screening. The study aimed to determine the fastest and the most effective auditory brainstem response measurement protocol in audiological diagnosis in babies up to three months of age.

\section{Methods}

\section{Participants}

The sample of the study comprised of newborns who were admitted to the Audiology Clinic from January 2015 through March 2019 and had passed the newborn hearing screening (NHS). Newborns were randomly selected from among the patients in the Audiology Clinic. The approval for the research was obtained from the Ethics Committee of Hacettepe University on March 18, 2015 (Approval number: GO 15/215-33) This study was carried out in compliance with the principles of the Helsinki Declaration. An informed consent form was obtained from the newborns' parents.

Patient evaluation forms including the demographic data of the newborns (age, sex, prenatal, perinatal, and postnatal history, and NHS scores) were filled-out by the parents. Twenty-two newborns (14 girls and eight boys) with a chronological age ranging from 0 to 63 days who had passed the NHS in at least one ear were examined in the study. Four of the examined newborns were followed for unilateral hearing loss and only the healthy ear that passed the NHS was evaluated in the study (Table 1).

\section{Main Points}

- In the single channel ABR systems, nape of the neck-vertexcheek electrode montages are more advantageous and comfortable than mastoid-mastoid-vertex electrode montages for both audiologists and newborns.

- For single channel ABR systems, different montage types had no effect on peak latencies.

- ABR peak latencies decrease with the lower intensity level regardless of the montage type.

\section{Inclusion Criteria}

Inclusion criteria of the study were as follows:

- a chronological age ranging from 0 to 63 days

- absence of a diagnosed syndrome that could lead to hearing loss

- absence of diagnosed developmental and/or neurological problems

- having passed (at least in one ear) the automatic ABR test performed within the scope of NHS

- normal otoscopic examination

- bilateral Type A tympanogram

\section{Exclusion Criteria}

Exclusion criteria of the study were:

- presence of any diagnosed developmental and/or neurological problem

- presence of any diagnosed syndrome that could lead to hearing loss

presence of any active middle ear pathology

\section{Auditory Brainstem Response (ABR)}

The ABR device used in the present study was Vivosonic ${ }^{\mathrm{TM}}$ Integrity ABR System (Toronto, ON, Canada), which is a single-channel device that utilizes bluetooth. For each newborn, five self-adhesive disposable electrodes and two foam probe tips in sizes suiting the ear-canal diameter were used. After the area where the electrodes would be placed was cleaned with cleansing gel to reduce electrical artefacts, the electrodes were placed before the newborn fell asleep. Following this procedure, the newborn was placed in a comfortable swing to help her/him sleep.

The measurements were performed using two different electrode montages (Figure 1). In the first montage, the negative electrode was placed on the ipsilateral mastoid, the ground electrode on the contralateral mastoid and the positive electrode on the vertex. In the second montage, the negative electrode was placed on the nape of the neck, the ground electrode on the cheek and the positive electrode on the vertex. Following the electrode placement, impedance values of all electrodes were checked. It was ensured that the impedance of each electrode was lower than $5 \mathrm{~kW}$ for the impedance measurement. The test time for each trace was determined using the same stopwatch in both montages. The electrode montage was selected randomly for each newborn.

The filter bandwidth used for recording was determined as 100$3000 \mathrm{~Hz}$. For the ABR measurement, a click stimulus was used and the recording was made at $70 \mathrm{dBnHL}, 50 \mathrm{dBnHL}$ and 20 $\mathrm{dBnHL}$ intensities, respectively. Ipsilateral recording was employed by taking two traces for each intensity. The repetition rate per second was determined as $27.5 \mathrm{msec}$ for both stimuli, and the recordings were made with a total stimulus repetition rate of 2,000 in rarefaction polarity. The response window was adjusted to be $0-20 \mathrm{msec}$. The traces that could not be analyzed reliably because of stimulus artefacts were not included in the study. The recordings were analyzed by experienced audiologists, and latencies for waves I, III and V, as well as test times were recorded. 
Table 1. General characteristics of newborns

\begin{tabular}{|c|c|c|c|c|c|}
\hline No & Gender & $\begin{array}{l}\text { Chronological age } \\
\text { (days) }\end{array}$ & Right ear NHS & Left ear NHS & $\begin{array}{l}\text { Evaluated ear } \\
\text { (ABR) }\end{array}$ \\
\hline 1 & Girl & 28 & Pass & Pass & Bilateral \\
\hline 2 & Girl & 30 & Pass & Pass & Bilateral \\
\hline 3 & Girl & 24 & Refer & Pass & Left \\
\hline 4 & Boy & 20 & Pass & Pass & Bilateral \\
\hline 5 & Boy & 8 & Pass & Pass & Bilateral \\
\hline 6 & Boy & 60 & Pass & Pass & Bilateral \\
\hline 7 & Girl & 28 & Pass & Pass & Bilateral \\
\hline 8 & Boy & 55 & Pass & Pass & Bilateral \\
\hline 9 & Boy & 52 & Pass & Pass & Bilateral \\
\hline 10 & Girl & 47 & Refer & Pass & Left \\
\hline 11 & Girl & 63 & Pass & Refer & Right \\
\hline 12 & Girl & 55 & Pass & Refer & Right \\
\hline 13 & Girl & 49 & Pass & Pass & Bilateral \\
\hline 14 & Girl & 59 & Pass & Pass & Bilateral \\
\hline 15 & Girl & 14 & Pass & Pass & Bilateral \\
\hline 16 & Boy & 22 & Pass & Pass & Bilateral \\
\hline 17 & Boy & 8 & Pass & Pass & Bilateral \\
\hline 18 & Boy & 26 & Pass & Pass & Bilateral \\
\hline 19 & Girl & 54 & Pass & Pass & Bilateral \\
\hline 20 & Boy & 54 & Pass & Pass & Bilateral \\
\hline 21 & Girl & 45 & Pass & Pass & Bilateral \\
\hline 22 & Girl & 63 & Pass & Pass & Bilateral \\
\hline
\end{tabular}

NHS: newborn hearing screening; ABR: auditory brainstem response

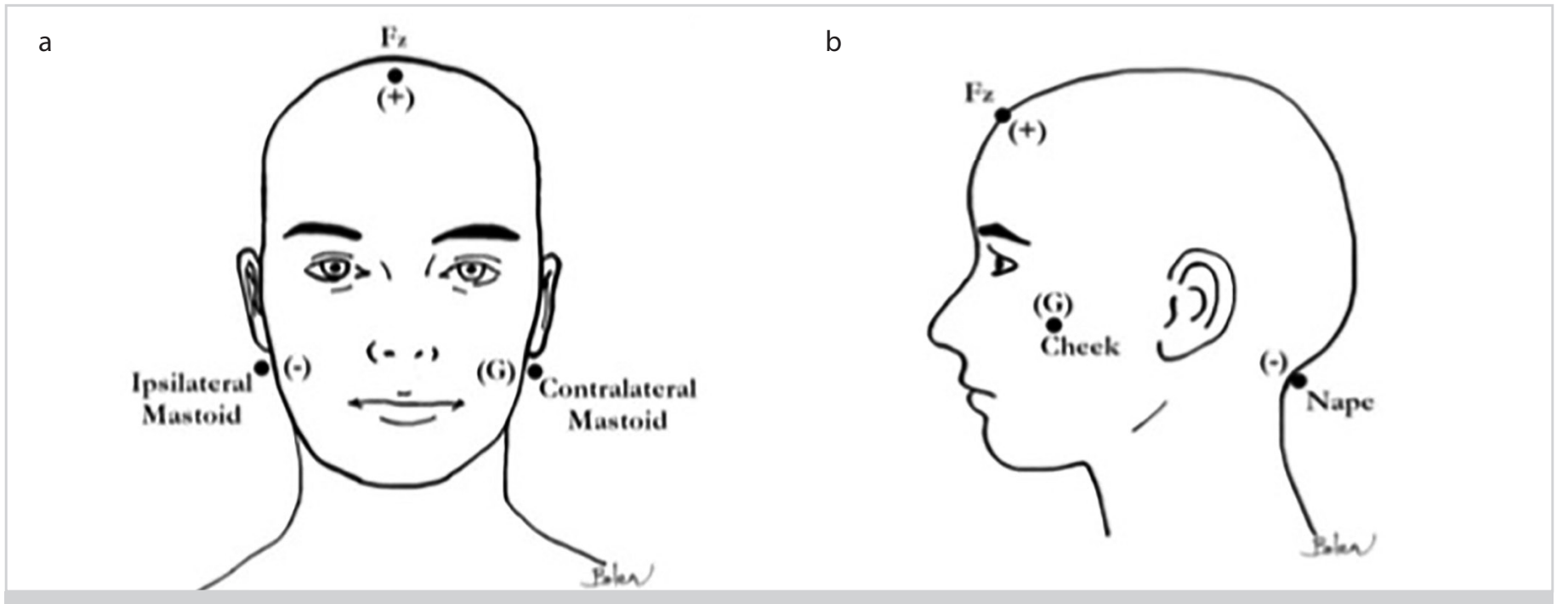

Figure 1. Electrode placement for ABR (a) $1^{\text {st }}$ electrode montages (b) $2^{\text {nd }}$ electrode montages

All measurements were carried out during sleep, and in cases when the newborn woke up, both the test and the stopwatch were paused until they fell asleep again.

\section{Statistical Analysis}

Statistical analysis was performed using IBM Statistical Package for the Statistical Package for the Social Sciences, PASW Statis- tics for Windows version 18.0 (SPSS Inc.; Chicago, IL, USA). In descriptive analyses, mean and standard deviation were used for numeric variables, while frequency distribution was used for ordinal variables. Because the number of subjects were less than 30 and that they did not show a normal distribution, the obtained data were evaluated using the non-parametric Mann-Whitney U test. Statistical significance level was accepted as $\mathrm{p}<0.05$. 


\section{Results}

\section{Descriptive Statistics}

The sample of the study consisted of 22 babies (14 girls and eight boys) 10 of which were younger than 30 days and the other 12 were older than 30 days. In total, 40 ears (20 left and 20 right ears) were examined in the study.

For each baby, the ABR test was performed using two different electrode montages as described in the methods section. Wave $\mathrm{V}$ latencies of ABR at $70 \mathrm{dBnHL}, 50 \mathrm{dBnHL}$ and $20 \mathrm{dBnHL}$, and the duration of recordings were compared for both montages.

\section{Comparison of Wave Latencies}

As it can be seen in Table 2, when the electrode montage was in its first state, wave $V$ latency was $6.86 \mathrm{msec}( \pm 0.50), 7.34 \mathrm{msec}$ $( \pm 0.43)$ and $8.44 \mathrm{msec}( \pm 0.66)$ at $70 \mathrm{dBnHL}, 50 \mathrm{dBnHL}$ and $20 \mathrm{dBnHL}$, respectively. In the second state, wave $V$ latency was $6.84 \mathrm{msec}( \pm 0.53), 7.36 \mathrm{msec}( \pm 0.63)$ and $8.43 \mathrm{msec}( \pm 0.62)$ at $70 \mathrm{dBnHL}, 50 \mathrm{dBnHL}$ and $20 \mathrm{dBnHL}$, respectively. It was seen that wave $\mathrm{V}$ latencies obtained from both electrode montages were the shortest at $70 \mathrm{dBnHL}$ and the longest at $20 \mathrm{dBn}$ HL. These differences were found to be statistically significant $(\mathrm{p}=0.00)$.
Statistical analyses showed that the recordings made at three intensities in two montages were not significantly different from each other in terms of their wave latencies (for $70 \mathrm{~dB} p=0.966$; for $50 \mathrm{~dB} p=0.475$; for $20 \mathrm{~dB} p=0.341 ; p>0.05$ ). Similarly, when the wave latencies of the babies aged 1 to 30 days and 31 to 63-days were compared at the same intensity levels, it was seen that there was no statistically significant difference between the two groups (for $70 \mathrm{~dB} p=0.180$; for $50 \mathrm{~dB} p=0.121$; for $20 \mathrm{~dB}$ $\mathrm{p}=0.198)$.

\section{Comparison of Test Times}

Table 3 shows the distribution of the times during which the waves recorded at the same intensity level in both montages were obtained. In the first montage type, the average recording times at $70 \mathrm{dBnHL}, 50 \mathrm{dBnHL}$, and $20 \mathrm{dBnHL}$ stimulation were $81.62( \pm 9.22), 88.41( \pm 19.64)$ and $82.38( \pm 11.67)$ seconds, respectively. In the second type, the average recording times at $70 \mathrm{dBnHL}, 50 \mathrm{dBnHL}$, and $20 \mathrm{dBnHL}$ stimulation were 85.89 $( \pm 13.21), 85.04( \pm 10.18)$ and $82.38( \pm 11.67)$ seconds, respectively. Statistical analysis comparing the recording times for the two montage types at three different intensities found that only the recording times at $50 \mathrm{dBnHL}$ showed a statistically significant difference $(\mathrm{p}=0.017)$. No statistically significant differences were observed in total test times and between the two electrode

Table 2. Wave V latencies

\begin{tabular}{l|c|c|c|c|c|c}
\hline \multicolumn{3}{|c|}{$\mathbf{1}^{\text {st }}$ electrode montages } & \multicolumn{2}{c}{$2^{\text {nd }}$ electrode montages } \\
\hline Stimulation level & N & Mean \pm SD (msec) & Min-Max (msec) & N & Mean $\pm S D(m s e c)$ & Min-Max (msec) \\
\hline $70 \mathrm{dBnHL}$ & 39 & $6.86 \pm 0.50$ & $6.10-8.44$ & 28 & $6.84 \pm 0.53$ \\
\hline $50 \mathrm{dBnHL}$ & 39 & $7.34 \pm 0.43$ & $6.46-8.13$ & 27 & $7.36 \pm 0.63$ \\
\hline $20 \mathrm{dBnHL}$ & 40 & $8.44 \pm 0.66$ & $7.14-9.95$ & 28 & $8.43 \pm 0.63$ \\
\hline
\end{tabular}

SD: standard deviation; Min: minimum; Max: maximum; msec: milliseconds; $\mathrm{dBnHL}$ : $\mathrm{dB}$ for normal hearing level

Table 3. Comparison of test times

\begin{tabular}{l|c|c|c|c|c|c|}
\hline \multicolumn{3}{|c|}{$1^{\text {st }}$ electrode montages } & \multicolumn{2}{c}{$2^{\text {nd }}$ electrode montages } \\
\hline Stimulation level & N & Mean \pm SD $(\mathbf{m s e c})$ & Min-Max (msec) & N & Mean \pm SD (msec) & Min-Max (msec) \\
\hline $70 \mathrm{dBnHL}$ & 40 & $81.62 \pm 9.22$ & $52-98$ & 28 & $85.89 \pm 13.21$ \\
\hline $50 \mathrm{dBnHL}$ & 39 & $88.41 \pm 19.64$ & $51-175$ & 27 & $85.04 \pm 10.18$ \\
\hline $20 \mathrm{dBnHL}$ & 39 & $82.38 \pm 11.67$ & $61-112$ & 27 & $82.38 \pm 11.67$ \\
\hline
\end{tabular}

SD: standard deviation; Min: minimum; Max: maximum; msec: milliseconds; $\mathrm{dBnHL}$ : $\mathrm{dB}$ for normal hearing level

Table 4. Comparison of the duration of test recordings (babies aged 1 to 30 days and 31 to 63 days)

\begin{tabular}{|c|c|c|c|c|c|c|c|}
\hline & \multirow{2}{*}{$\begin{array}{c}\text { Stimulation } \\
\text { level }\end{array}$} & \multicolumn{3}{|c|}{$1^{\text {st }}$ electrode montages } & \multicolumn{3}{|c|}{$2^{\text {nd }}$ electrode montages } \\
\hline & & $\mathrm{N}$ & Mean \pm SD (msec) & Min-Max (msec) & $\mathbf{N}$ & Mean \pm SD (msec) & Min-Max (msec) \\
\hline \multirow[t]{2}{*}{1 to 30 days } & $70 \mathrm{~dB}$ & 19 & $85.94 \pm 6.46$ & $77-98$ & 16 & $89.81 \pm 15.35$ & $75-137$ \\
\hline & $20 \mathrm{~dB}$ & 18 & $83.84 \pm 9.75$ & $66-105$ & 16 & $83.82 \pm 7.12$ & $75-97$ \\
\hline \multirow[t]{2}{*}{31 to 63 days } & $70 \mathrm{~dB}$ & 21 & $77.90 \pm 9.73$ & $52-90$ & 12 & $80.67 \pm 7.4$ & $71-91$ \\
\hline & $20 \mathrm{~dB}$ & 21 & $81.05 \pm 13.26$ & 61-112 & 11 & $87.64 \pm 16.87$ & $68-119$ \\
\hline
\end{tabular}


placements at other intensity levels (for $70 \mathrm{~dB} p=0.553 ; 20 \mathrm{~dB}$ $\mathrm{p}=0.483$ ).

When the test times at the same intensity levels in babies aged 1 to 30 days and 31 to 63 days were compared for two montages (Table 4$)$, there were statistically significant $(p=0.005)$ differences between the test times recorded at $70 \mathrm{dBnHL}$. There were, however, no statistically significant differences between the other two intensity levels (for $50 \mathrm{~dB} \mathrm{p}=0.075$; for $20 \mathrm{~dB} \mathrm{p}=0.399$ ).

\section{Discussion}

The main result of our study is the comparability of the latencies found in two different electrode montages. In regard of our results, the use of the same montage in single channel ABR devices can help to perform more useful testing in newborns.

As it is an objective method that does not require patient participation, $A B R$ testing is often used for assessing hearing in populations with cooperation problems. Accordingly, it is a very convenient test that shows the integrity of the auditory pathway and helps to estimate hearing thresholds. Many protocol manuals for hearing assessment in pediatric groups, ABR is addressed as a very important part of the test battery. The aim of this study was to assess whether or not two electrode montages are different in terms of ABR test results, and to determine which electrode montage is more advantageous.

Studies reported in the literature mention that latencies decrease with age and increase with decreased stimulus amplitude $(1,11)$. Consistent with the reports in the literature, in our study we observed that babies aged 1 to 30 days had significantly prolonged wave $V$ latencies compared to those aged 31 to 63 days. Moreover, latencies at $20 \mathrm{dBnHL}$ were significantly prolonged compared to $70 \mathrm{dBnHL}$.

Previous studies have shown different electrode montage types and mentioned that different montages can change ABR wave form morphology. Vertical montage came forth in past decade and was frequently used. Vertical montage is different than standard ipsilateral montage, particularly in terms of the negative electrode that is attached to the non-cephalic back of the neck. In classic ipsilateral $A B R$, electrodes are attached to both the mastoids and the forehead, and click stimulus is used. ABR studies using click stimulus mention that electrodes attached to the back of the neck, the forehead and the vertex (vertical montage) can provide artefact-free, large amplitude ABR waves (69\% larger compared to standard mastoid montage) (12-14). In our study, no effect of electrode montage on wave $V$ latencies were observed at any stimulus intensity. Likewise, this effect was also not observed when recordings were compared between the 1 to 30 -day-old and the 31 to 63 -day-old groups.

One of the aims of our study was to investigate the possible effects of different electrode montages on test time- to our knowledge, no previous study has investigated this aspect. Comparison of test times at different intensity levels found that the first montage required longer test times at $50 \mathrm{dBnHL}$ stimulus intensity. In the light of this finding, the second montage can be suggested for a shorter test time in tests at the medium intensity level (50 dBnHL). When, however, test times were compared based on age at $70 \mathrm{dBnHL}$ intensity level, the test times of the first montage were significantly shorter in the 31 to 63 -day-old group than the 1 to 30 -day-old group. The effect of the montage type on the test time is an important information when completing the test reliably within a short time is of the essence. Further studies are needed to systematically test this effect.

In our study, we saw that babies had different sleeping timessomething that can negatively affect the test or hinder its optimal completion. Arrangements (using dim light, using a swing, etc.) were made before the test to eliminate environmental impact. Nevertheless, there was loss of data because babies did not sleep throughout the test. Not all tests of all newborns could be completed. This is an important drawback of the study. Another main limitation of our study could be the absence of the amplitudes of each wave compared.

In single channel $A B R$ devices the place of the reference electrodes has to be changed for each ear. This can wake the baby up during the test. This study has shown that electrode montage does not lead to differences in wave latencies and total test times. Placing the electrodes so as not to disturb the baby is very important in terms of practicality. Completing the ABR, which is of great significance for early diagnosis, reliably in a short time is very important. Investigation of this issue can conclude that, in terms of practicality, using the second montage which is done once without requiring the electrodes to be re-placed is both advantageous for the audiologist and comfortable for the newborn.

To summarize, in line with general knowledge; ABR peak latencies decrease regardless of the montage type. In fact, different montage types had no effect on peak latencies. An effect of the montage type was observed on test time at medium intensity level $(50 \mathrm{dBnHL})$, where the test time for the second montage was shorter, but no difference was observed at the other intensity levels. When the two groups of babies were compared in terms of the effect of the montage type on the test time, the first montage type was significantly shorter in 31 to 63-day-old babies compared to 1 to 30 -day-olds. Further studies are needed to systematically investigate the effects of montage types on test time. Keeping in mind that our findings are from normal hearing babies, another suggestion may be to conduct similar comparisons on babies with hearing loss at different degrees for exploring whether or not similar effects do exist.

Ethics Committee Approval: Ethics committee approval was received for this study from the Ethics Committee of Hacettepe University (Approval Date: March 18, 2015; Approval Number: GO $15 / 215-33)$.

Informed Consent: Informed consent was obtained from the parents of the patients who participated in this study.

Peer-review: Externally peer-reviewed. 
Author Contributions: Concept - G.S.; Design - G.S.; Supervision G.S.; Materials - Ö.Y., M.Ö.B., B.Ç.Ç., M.Y., M. Yaralı; Data Collection and/or Processing - Ö.Y., M.Ö.B., B.Ç.Ç., M.Y., M. Yaralı; Analysis and/or Interpretation - B.Ç.Ç.; Literature Search - Ö.Y., M.Y.; Writing - Ö.Y., M.Ö.B., M. Yaralı; Critical Reviews - G.S.

Conflict of Interest: The authors have no conflicts of interest to declare.

Financial Disclosure: The authors declared that this study was supported by Hacettepe University Scientific Research Projects Coordination Unit (Project Number:TAY-2015-6423).

\section{References}

1. Hyde M. Principles and methods of population hearing screening in EHDI. Tharpe AM and Seewald R, editors. Comprehensive Handbook of Pediatric Audiology. San Diago: Plural Publishing Inc.; 2011. p. 283-336.

2. Hahn M, Lamprecht-Dinnesen A, Heinecke A, Hartmann S, Bülbül S, Schröder G, et al. Hearing screening in healthy newborns: feasibility of different methods with regard to test time. Int J Pediatr Otorhinolaryngol 1999; 51: 83-9. [Crossref]

3. Thompson DC, McPhillips H, Davis RL, Lieu TA, Homer CJ, Helfand M. Universal newborn hearing screening: summary of evidence. JAMA 2001; 286: 2000-10. [Crossref]

4. Bolat H, Genç GA. Türkiye ulusal yenidoğan işitme taraması programı: tarihçesi ve prensipleri. Turkiye Klinikleri J E.N.T. 2012; 5:11-4.

5. Hall JW 3rd, Smith SD, Popelka GR. Newborn hearing screening with combined otoacoustic emissions and auditory brainstem responses. J Am Acad Audiol 2004; 15: 414-25. [Crossref]
6. Stewart DL, Mehl A, Hall JW 3rd, Thomson V, Carroll M, Hamlett J. Universal newborn hearing screening with automated auditory brainstem response: a multisite investigation. J Perinatol 2000; 20: 128-31. [Crossref]

7. Atcherson SR, Stoody TM, editors. Auditory electrophysiology: a clinical guide. New York: Thieme; 2012; 9-24.

8. Stevens J, Brennan S, Gratton D, Campbell M. ABR in newborns: effects of electrode configuration, stimulus rate, and EEG rejection levels on test efficiency. Int J Audiol 2013; 52: 706-12. [Crossref]

9. Dzulkarnain AAA, Noor Ibrahim SHM, Anuar NFA, Abdullah SA, Tengku Zam TZH, Rahmat S, et al. Influence of two-electrode montages on the level-specific (LS) CE-Chirp auditory brainstem response (ABR) at multiple intensity levels. Int J Audiol 2017; 56: 723-32. [Crossref]

10. Keesling DA, Parker JP, Sanchez JT. A comparison of commercially available auditory brainstem response stimuli at a neurodiagnostic intensity level. Audiol Res 2017; 7:161. [Crossref]

11. Öztürk B, Genç GA. Maturation of auditory brainstem responses in babies from birth to 6 months of age. Turkiye Klinikleri J Med Sci 2012; 32: 677-86. [Crossref]

12. Hall JW. New handbook of auditory evoked responses. 3rdh ed. Boston: Pearson; 2007; 71-211.

13. Dzulkarnain AA, Wilson WJ, Bradley AP, Petoe M. The effects of electrode montage on the amplitude of wave $\mathrm{V}$ in the auditory brainstem response to maximum length sequence stimuli. Audiol Neurotol 2008; 13: 7-12. [Crossref]

14. Dzulkarnain AAA, Buyong AS, Sulaiman NH. Intra-subject variability in the auditory brainstem response using a vertical montage recording. Speech Lang Hear 2014; 17: 160-7. [Crossref] 